level. There was evidence that the increased confidence had been sustained.

This project demonstrates how the hospice is engaging with it's local community to build capacity and better equip everyone in supporting young people experiencing loss and bereavement.

\section{P141 A REVIEW OF THE MULTI DISCIPLINARY TEAM'S (MDT) PERCEPTION OF THE PSYCHOSOCIAL SERVICES TEAM'S (PST) INTERVENTION ON THE IN PATIENT UNIT (IPU)}

Martin Riley. Hospice in the Weald, Pembury, United Kingdom

\subsection{6/bmjspcare-2013-000591.163}

This project considered how counselling is offered, accessed, received and perceived on the In-Patient Unit (IPU) at Hospice in the Weald. Questions were formed about adapting and modifying the existing service to better meet the needs of our service users.

Unstructured interviews were held with professional MDT groups, inviting them to discuss perceptions of psychosocial care and how patients' needs are met on the IPU. Sessions were audio recorded, transcribed and analysed.

A new model of care was proposed. Counsellors rotated through IPU for 6 weeks. At the end of the rotations, each counsellor was interviewed, as was the larger MDT (in one collective group). Interviews were recorded and salient learning points identified. After each rotation, questionnaires were given to IPU staff to identify the benefits, things to change and give feedback.

We learned that traditional methods of referral, assessment and practice of counselling are inappropriate to the specialist setting of in-patient care. Questions about ownership of psychosocial care, referral processes, multidisciplinary aspects of collaborative working, audit/review and IPU admissions for psychological care were asked and partially answered in the study. Interviewing staff after the change in practice revealed much more satisfaction with the counselling service and many advantages arising from the change in practice. Improved MDT working and speed of access to counselling were cited most frequently.

The wider implications include considering how we modify our practice to better meet the needs of our service users. Working differently may lead to investigations about what we call this approach to the provision of psychological support to in-patients and their relatives. For some it's still counselling, for others it's an exciting challenge to expand both their practice and capacity to offer support to those facing the end of a life; particularly those who otherwise reject talking therapy.

\section{P142 DISCUSSING PREFERRED PLACE OF DEATH WITH PATIENTS: STAFF EXPERIENCE IN A PALLIATIVE CARE SETTING}

${ }^{1,2}$ Anna Fields, Anne Finucane, 'David Oxenham. 'The University of Edinburgh, Edinburgh, United Kingdom, ${ }^{2}$ Marie Curie Hospice, Edinburgh

\subsection{6/bmjspcare-2013-000591.164}

Aims To qualitatively explore staff members' experiences of discussing preferred place of death (PPD) with patients at the Marie Curie Hospice Edinburgh (MCHE).

Introduction National end-of-life care policies propose that health-care professionals should have regular end-of-life discussions with patients wishing to talk about such issues. In 2007 the process of identifying patient end-of-life preferences was formalised at the MCHE; and staff now routinely discuss and record PPD. This study explores staff experiences of these discussions.

Method Six members of medical and nursing staff were recruited from the inpatient, day-service and community teams at the MCHE. Participants took part in an in-depth, audiorecorded, semi-structured interview. Interviews were transcribed verbatim and analysed using Interpretative Phenomenological Analysis (IPA), an approach to phenomenology which aims to capture and understand lived experience.

Results Four themes were identified as being integral to participants' accounts: "The importance of discussing preferences at the end of life"- staff recognise the importance of discussing and trying to achieve patients' final wishes; "Identifying how and when to discuss PPD"- staff identify cues from patients to ensure discussions occur at a time and pace suitable for the individual; "Reflecting on the emotional aspects of discussing PPD"- discussing PPD is challenging but important and rewarding; "A journey from expectations to experience"- discussing PPD becomes easier over time through practice and learning from others.

Conclusion These findings highlight that although PPD discussions can be difficult, staff feel that discussing future plans, including place of death, with patients is important and beneficial for both patients and their families. With time, staff members have developed communication strategies that allow them to discuss PPD in an effective, sensitive and patient-centred way.

\section{P143 MEMORIES MATTER: FACILITATING EXPRESSION THOUGH CREATIVITY}

Debbie Ho, Andrea Davies, Val Bailey, Susie Carr, Sarah Maxwell, Charlie Rendell. Prospect Hospice, Swindon, UK

\subsection{6/bmjspcare-2013-000591.165}

Introduction In 2012 the Hospice extended the reach of its traditional family support services with two dedicated events for children and families. Each event was designed to facilitate remembrance, and promote self care and confidence within families whose lives had been inextricably changed though loss.

Aim To create a bespoke event for children and adults (both) independently and collectively through providing a range of creative and therapeutic activities.

To test simple strategies for engagement and interaction, and evaluate the quality of the experience through simple discovery techniques.

To reduce the sense of isolation experienced by children following the loss of someone loved by them.

Method 28 children in total attended the events which took place in July and November 2012. Led by the family support and bereavement care service, and supported by the art therapist and trained volunteers, children and families who had previously engaged in pre-bereavement support, or post bereavement 1:1 or small group support were invited to the events.

A range of activities were developed in consultation with the art therapist with a view to engaging children across a broad range of ages. Some sought to unify children and adult involvement. Activities included:

- Making memory felts

- Making a web of support using a ball of wool to create a shared bond

- Making an interactive advent calendar which featured on the Hospice website throughout December creating a meaningful focus as families faced a first Christmas. 